\title{
Rabies vaccine potency control: comparison of ELISA systems for antigenicity testing
}

\author{
E.J.M. Rooijakkers ${ }^{\mathrm{a}}$, J.P. Uittenbogaard ${ }^{\mathrm{b}}$, J. Groen ${ }^{\mathrm{a}}$, A.D.M.E. Osterhaus ${ }^{\mathrm{a}, \mathrm{c}, *}$ \\ a Department of Clinical Virology, University Hospital Rotterdam, Dr. Molewaterplein 40, 3015 GD Rotterdam, The Netherlands \\ ${ }^{b}$ Laboratory of Vaccine Development and Immune Mechanisms, National Institute of Public Health and Environmental Protection, \\ P.O. Box 1, 3720 BA Bilthoven, The Netherlands \\ 'Institute of Virology, Erasmus University Rotterdam, P.O. Box 1738, 3000 DR Rotterdam, The Netherlands
}

Accepted 6 December 1995

\begin{abstract}
Potency control of inactivated rabies vaccines for human and veterinary application is usually undertaken by vaccination-challenge tests (e.g. the mouse potency test). For practical and ethical reasons there is an urgent need to replace in vivo potency control procedures, at least in part, by reliable methods of in vitro potency testing. Quantitative ELISA systems for potency control were developed using monoclonal antibodies (MAbs) directed to the glycoprotein (GP) and to the nucleoprotein (NP) of the virus. Although immuno-capture and competition ELISAs for GP measurement had almost equal sensitivity (detection level GP $<0.1$ IU), the competition data showed the best correlation with potency values when a panel of rabies vaccines for human use was tested $(r=0.88, n=10)$. The NP values for this panel of vaccines in the competition system (detection level NP $<1$ IU) also correlated well with potency values $(r=0.90, n=10)$. The competition system proved to be best also with liquid adjuvanted veterinary rabies vaccines of LEP, PM, PV and SAD origin. The implementation of ELISA systems for potency control of rabies vaccines is discussed.
\end{abstract}

Keywords: Rabies vaccine; Potency assay; Antigenicity; ELISA

\section{Introduction}

According to the recommendations of the World Health Orgenization (WHO, 1992) and the European Pharmacopoeia (1985), potency control of rabies vaccines for human and animal use is carried out generally by mouse potency tests (e.g. the NIH potency test) (Seligmann, 1973). How-

\footnotetext{
* Corresponding author. Tel.: + 3110 4088066; fax: + 31 104365145 .
}

ever, this laborious in vivo vaccination-challenge procedure in mice is subject to poor reproducibility, mainly due to hetcrogeneity in mice and challenge procedures used (Barth et al., 1988). For both practical and ethical reasons, replacement of in vivo potency tests by more rapid and more reliable in vitro methods for potency control is highly desirable. In spite of the formal possibility of replacing the mouse potency test by in vitro antigenicity control methods (WHO, 1992), no such alternative assay is widely used at present. 
We placed emphasis on the development of ELISA procedures which meet the WHO requirements for potency control (WHO, 1992). Newly developed monoclonal antibody-based ELISA systems are described for direct quantification of the glycoprotein (GP) and nucleoprotein (NP), which constitute the major immunogenic components of rabies vaccines. The results of antigenicity testing of a panel of dog kidney cell-culture rabies vaccines by the ELISAs are described and the correlation with potency values obtained in the NIH potency test is evaluated. Furthermore, the application of the ELISAs for testing the antigenicity of veterinary vaccines of different origins with different adjuvants is evaluated.

\section{Materials and methods}

\subsection{Rabies vaccines}

Freeze-dried inactivated human rabies vaccines of the Pitman-Moore strain propagated on primary dog kidney cells (PM-DKC), produced routinely at the National Institute of Public Health and Environmental Protection (RIVM, Bilthoven, The Netherlands), and commercial liquid inactivated (cell culture) veterinary rabies vaccines of various origin (strains PV, SAD, PM, Flury-LEP) were stored in a refrigerator until use.

The 5th International Standard for Rabies Vaccine (Lyng et al., 1992) containing 16 international units (IU) of rabies vaccine (potency value $16 \mathrm{IU}$ ), $10 \mathrm{IU}$ of rabies PM-glycoprotein (GP value $10 \mathrm{IU}$ ) and 135 IU of rabies PM-nucleoprotein (NP value 135 IU) per ampoule, and the in-house RIVM reference rabies vaccine (R0-41A, potency value 4.2 IU), were used as reference for the NIH potency test and the ELISA systems developed in the course of these studies.

\subsection{Potency test}

The mouse potency test was carried out following a standardized operating procedure, essentially according to the WHO protocol for human rabies vaccines (NIH potency test, Seligmann, 1973). Briefly, SPF outbred NIH mice (strain Riv:NIH) (Hendriksen et al., 1994), were at the age of 4 weeks, distributed randomly in groups of 20 animals (one sex/group). Freshly prepared serial 5fold dilutions of rabies vaccines (samples and in-house reference) were used for intraperitoneal immunization ( $0.5 \mathrm{ml}$ doses) of mice on day 0 and day 7. On day 14 each mouse was challenged intracerebrally with $30 \mu 1$ rabies virus CVS-26 and observed daily during 2 weeks for the development of rabies symptoms.

The challenge dose $\left(\mathrm{LD}_{50}\right)$ of CVS-26 was titrated in control mice and calculated according to the procedure of Reed and Muench (Lorenz and Bögel, 1973). The protective doses $\left(\mathrm{ED}_{50}\right)$ were calculated according to the Probit analysis of Bliss (Bliss, 1935). Vaccine potencies were expressed in International Units rabies vaccine (IU RV).

\subsection{Monoclonal antibodies}

Spleen cells isolated from BALB/c mice, immunized previously with rabies vaccine produced on dog kidney cells, were fused with myeloma cells (P3-X63-Ag8.653 or $\mathrm{Sp} 2 / 0$ ) to generate murine hybridomas, as described previously (Osterhaus et al., 1982). Rabies virus-specific antibody secreting hybridomas were selected by ELISA using PM virus antigen coated microtiter plates. Single cell cloned hybridomas were ultimately propagated in vitro. The monoclonal antibodies (MAbs) were purified from the cell culture fluids by immunoaffinity chromatography on a Protein A or Protein G sepharose CL-4-B column (Pharmacia, Uppsala, Sweden). Eluted Ig was concentrated by the precipitation method with saturated ammonium sulphate, essentially as described previously (Hong et al., 1994). Murine glycoprotein-specific virus neutralizing MAbs (GP-MAb) 2-22 and 6-15 have been described previously (Bunschoten et al., 1989), whereas 78-9 was developed in the course of this study as described above.

Human hybridomas, secreting NP-specific MAb (NP-MAb) 56 and 105, kindly provided by Dr. P. Casali (Department of Pathology, New York University Medical School, New York, USA), were generated from EBV-transformed peripheral B lymphocytes (Dietzschold et al., 1990). The human MAbs 56 and 105 were produced and purified as described above for murine MAbs. 
MAbs were conjugated to horseradish peroxidase (HRPO) or to biotin (NHS-D-Biotin; Sigma Chemie), essentially according to methods described previously (Nakane and Kawaoi, 1974; Bayer and Wilchek, 1980).

\subsection{ELISA: standard procedure}

In general, ELISAs were carried out according to standardized operating procedures, resulting in optimum conditions for each type of test. Briefly, the coating procedure of ELISA plates was carried out with $100 \mu 1$ aliquots/well $\left(16 \mathrm{~h}, 4^{\circ} \mathrm{C}\right)$. Subsequently, the plates were washed in a semiautomatic washing device using de-ionized water containing detergent (Tween 20) and emptied. The solid phase was blocked with an excessive amount of irrelevant proteins (e.g. BSA) in PBS (150 $\mu \mathrm{l} /$ well, $1 \mathrm{~h}, 37^{\circ} \mathrm{C}$ ), to reduce non-specific reactions. Between the successive steps of sample and conjugate incubation $\left(100 \mu \mathrm{l} /\right.$ well, $\left.1 \mathrm{~h}, 37^{\circ} \mathrm{C}\right)$, the plates were washed and emptied. ELISA plates were developed by a TMB-based substrate solution (100 $\mu \mathrm{l}$ per well) and the reaction was stopped after $10 \mathrm{~min}$ by addition of sulphuric acid, as described previously (Groen et al., 1989). The absorbance $\left(A^{\prime}\right)$ was measured at wavelength $450 \mathrm{~nm}\left(\mathrm{OD}_{450}\right)$, using a spectrophotometer for microtiter plates (Гitertek Multiscan).

\subsection{Characterization of MAbs by ELISAs}

\subsubsection{Identification of GP- and NP-producing hybridomas}

Mouse hybridoma cell lines producing antibodies to the rabies virus GP were identified with the Platelia Rabies kit (Sanofi Diagnostics), detecting antibodies to GP. Human hybridoma cell lines producing antibodies to the rabies virus NP were identified by indirect ELISA on NP-antigen coated ELISA plates. The NP-antigen, expressed by a recombinant baculovirus in insect cells, was kindly provided by Dr. F. Makkonen, Public Health Service, CIC, Lawrenceville, USA).

\subsubsection{Epitope mapping with GP- and NP-specific $M A b s$}

Competitive binding studies by antibody-inhibi- tion ELISAs were carried out to determine epitope and antigenic site specificity of different GPand NP-specific MAbs available, as described previously (Bunschoten et al., 1989). Briefly, ELISA plates were coated with a standard preparation of rabies virus antigen (PM-DKC vaccine), followed by incubation with a GP- or NP-specific MAb (inhibitor). Subsequently, the biotin conjugated $\mathrm{MAb}$, homologous or heterologous to the inhibitor, was incubated at the specified concentration which corresponded to $70 \%$ of the maximal absorbance $\left(A_{\max }\right)$ in control experiments. The reaction of the biotin conjugated $\mathrm{MAb}$ without inhibitor $\left(A_{\text {control }}\right)$ was included in the experiments as the control. The results of the inhibition ELISA were expressed as the percentage of inhibition ( $\%$ inhibition), according to the formula:

$\%$ inhibition $=\left(A_{\text {control }}-A_{\text {test }}\right) / A_{\text {control }} \times 100 \%$

\subsection{Antigen quantification by ELISAs}

\subsubsection{Immunocapture GP ELISA}

The immunocapture antibody-sandwich principle was used to develop quantitative assays for GP measurement, according to the procedure described previously (Osterhaus et al., 1990). Briefly, GP-MAb 6-15 or 78-9 (respectively for immunocapture GP ELISA 6-15 and immunocapture GP ELISA 78-9) was coated on microtiter plates, followed by blocking with irrelevant proteins. Subsequently, serial 2-fold dilutions of rabies vaccine were incubated, followed by incubation with biotin conjugated MAb 2-22. Subsequently, streptavidin-HRPO was allowed to bind.

\subsubsection{Competition GP and NP ELISA}

Antigen competition ELISAs for the quantitative determination of GP and NP contents in rabies vaccines were developed with GP-MAb 2-22 and NP-MAb 56 respectively. Briefly, serial 2-fold dilutions of rabies vaccine were incubated with a previously titrated amount of biotin conjugated MAb 2-22 or 56 in a low binding microtiter plate. Subsequently, the vaccine-MAb mixture was transferred to an ELISA plate coated with a standard preparation of rabies virus antigen (PM- 
DKC vaccine) to determine the remaining antigen binding capacity of the biotin conjugated MAb. After incubation and washing, streptavidinHRPO was allowed to bind.

\subsection{Calculation procedures for quantitative ELISA systems}

The GP and NP contents of vaccines are expressed in IU, with reference to the 5 th International Standard for Rabies Vaccine (Lyng et al., 1992), supplied by the WHO.

The antigenicity was calculated by a regression analysis method, essentially as described by Thraenhart and Ramakrishnan (1990). Briefly, a dose response curve for the reference was created by plotting the absorbance ( $\mathrm{Y}$-axis) as a function of the potency value (X-axis). The 'linear range' of the (sigmoidal) reference curve was identified and the equation for this range (reference line) was calculated by linear regression analysis and used to determine relative potency values of the samples. Finally, the relative potency value was multiplied by the sample dilution to calculate the antigenic value (IU) for GP or NP of the vaccine sample. The sensitivity of the respective ELISA systems was defined as the relative antigenic value for GP or NP determined half-way up the titration curve of the reference.

\section{Results}

\subsection{Selection of murine and human MAbs}

The study started with competitive binding ELISAs for epitope mapping of murine virus neutralizing GP-specific monoclonal antibodies (GP-MAb) and human NP-specific monoclonal antibodies (NP-MAb) being available. Three GPMAbs (2-22, 6-15 and 78-9) were selected which proved to recognize different epitopes on functionally independent antigenic sites of the rabies virus GP, respectively antigenic site I, VI and II/III. Two NP-specific MAbs (56 and 105) recognized different, but spatially related epitopes on the rabies virus NP. NP-MAb 56 was selected for further studies on account of its higher avidity in comparison to NP-MAb 105.

\subsection{Assessment of $G P$ and NP contents in $P M-D K C$ vaccines}

Quantification of GP contents in vaccines was undertaken by two immunocapture ELISAs (immunocapture GP ELISA 6-15 and immunocapture GP ELISA 78-9) and one competition ELISA (competitive GP ELISA), all using biotin conjugated GP-MAb 2-22 as detector antibody. NP contents in vaccines were quantitated by a competition NP ELISA using biotin conjugated NP-MAb 56 as detector antibody. Experimental conditions for the respective ELISA systems were optimized, which resulted in detection levels for GP of 0.03 IU in the immunocapture GP ELISA 6-15 and 0.05 IU in both the immunocapture GP ELISA 78-9 and the competition GP ELISA. The detection level for NP by the competition NP ELISA was $0.8 \mathrm{IU}$.

GP and NP values of ten consecutive production lots of PM-DKC vaccines were assessed by the respective ELISAs, using the International Standard for rabies vaccine as reference. As shown in Table 1, immunocapture GP ELISA 6-15 gives lower GP values than immunocapture GP ELISA 78-9 and competition GP ELISA, the latter two assays showing similar GP values.

Antigenic values for GP and NP by the different ELISAs all showed similar correlation with potency values established in NIH potency test (Fig. 1), correlation coeffcients $(r)$ ranged from 0.71 to 0.90 , as indicated in the graphs.

\subsection{Use of ELISA systems for veterinary rabies vaccines}

Seven commercial veterinary rabies vaccines were titrated by the respective ELISA systems for measurement of GP and NP contents (Fig. 2). One of the vaccines of SAD origin adjuvanted with a carbohydrate polymer (sample 1, Fig. 2) could not be evaluated by the different ELISAs: absorbancy (OD values) by immunocapture GP ELISAs 6-15 and 78-9 were out of range for regression analysis, whereas by competition ELISAs for GP and NP no parallelism was found between titration curves of sample 1 and reference. Furthermore, immunocapture GP ELISA 
Table 1

GP and NP values (IU) in different ELISAs for ten PM-DKC rabies vaccines

\begin{tabular}{|c|c|c|c|c|}
\hline \multirow[t]{2}{*}{ Vaccine lot no. } & \multicolumn{4}{|l|}{ Antigenic value } \\
\hline & Inmunocapture GP 6-15 & Immunocapture GP 78-9 & Competition GP & Competition NP \\
\hline 1 & 2.1 & 7.0 & 6.7 & 198 \\
\hline 2 & 13.4 & 26.8 & 26.8 & 718 \\
\hline 3 & 3.6 & 9.0 & 13.2 & 267 \\
\hline 4 & 5.2 & 14.3 & 20.2 & 443 \\
\hline 5 & 0.7 & 1.8 & 2.2 & 97 \\
\hline 6 & 3.4 & 8.5 & 10.5 & 372 \\
\hline 7 & 1.7 & 4.2 & 5.7 & 316 \\
\hline 8 & 13.2 & 22.4 & 42.5 & 1181 \\
\hline 9 & 10.3 & 23.5 & 28.0 & 1029 \\
\hline 10 & 13.5 & 35.6 & 36.0 & 1077 \\
\hline
\end{tabular}

6-15 could not be used for the LEP vaccine (sample 4) and one of the two PV vaccines (sample 5) in the panel tested. Coating of these vaccines in indirect ELISA with GP-MAb 6-15 proved that the respective vaccine strains were not recognized by the MAb (data not shown). The other ELISAs (immunocapture GP ELISA 78-9, competition GP ELISA and cornpetition NP ELISA) could be used for all vaccines of PM, PV, SAD (except sample 1) and LEP origin involved. Titration curves of these vaccines showed lines parallel to the titration curve of the International Standard for rabies vaccine by the respective ELISAs.

\section{Discussion}

In Europe, millions of human and veterinary rabies vaccine doses are produced annually by cell culture techniques (Sureau, 1992). These vaccines are used world-wide, with the exception of certain developing countries where traditional neural tissue vaccines are produced for local use. Released batches of these rabies vaccines should meet the international requirements for potency, specified by the WHO Expert Committee on Rabies (WHO, 1992). Potencies of these vaccines are usually determined in in vivo mouse potency tests, which require world-wide tens of thousands of mice annually, which must be challenged with virulent rabies virus.
There is a consensus amongst control authorities and manufacturers that a replacement or at least a refinement and reduction of such unethical animal tests should be sought. Furthermore, the clear practical drawbacks (e.g. laborious, timeconsuming and expensive procedures) and the poor intra- and inter-laboratory reproducibility of mouse potency tests are subject to substantial criticism (Barth et al., 1988). The incentive to replace the in vivo potency test is reflected by the efforts of numerous laboratories to introduce reliable alternative in vitro methods. This has, however, led to only limited numbers of proposals for actual replacement. So far, the modified antibody binding test (ABT) (Barth et al., 1981; Barth et al., 1990) and the single radial diffusion test (SRD) (Ferguson and Schild, 1982; Ferguson et al., 1984; Ferguson and Heath, 1992) were proposed as alternatives. Their use is restricted to GP measurement by polyclonal antibodies and is not applicable to adjuvanted vaccines. The ELISA systems described so far for potency estimation were all based on polyclonal antibodies against the GP or NP (Atanasiu et al., 1980; Van der Marel and Van Wezel, 1981; Thraenhart and Ramakrishnan, 1989; Perrin et al., 1990) or on MAbs specific for GP (Lafon et al., 1985). In our view, the methods of choice should measure antigenicity with GP- and NP-MAbs recognizing all vaccine strains and should be suitable for final lot control of adjuvanted vaccines. 

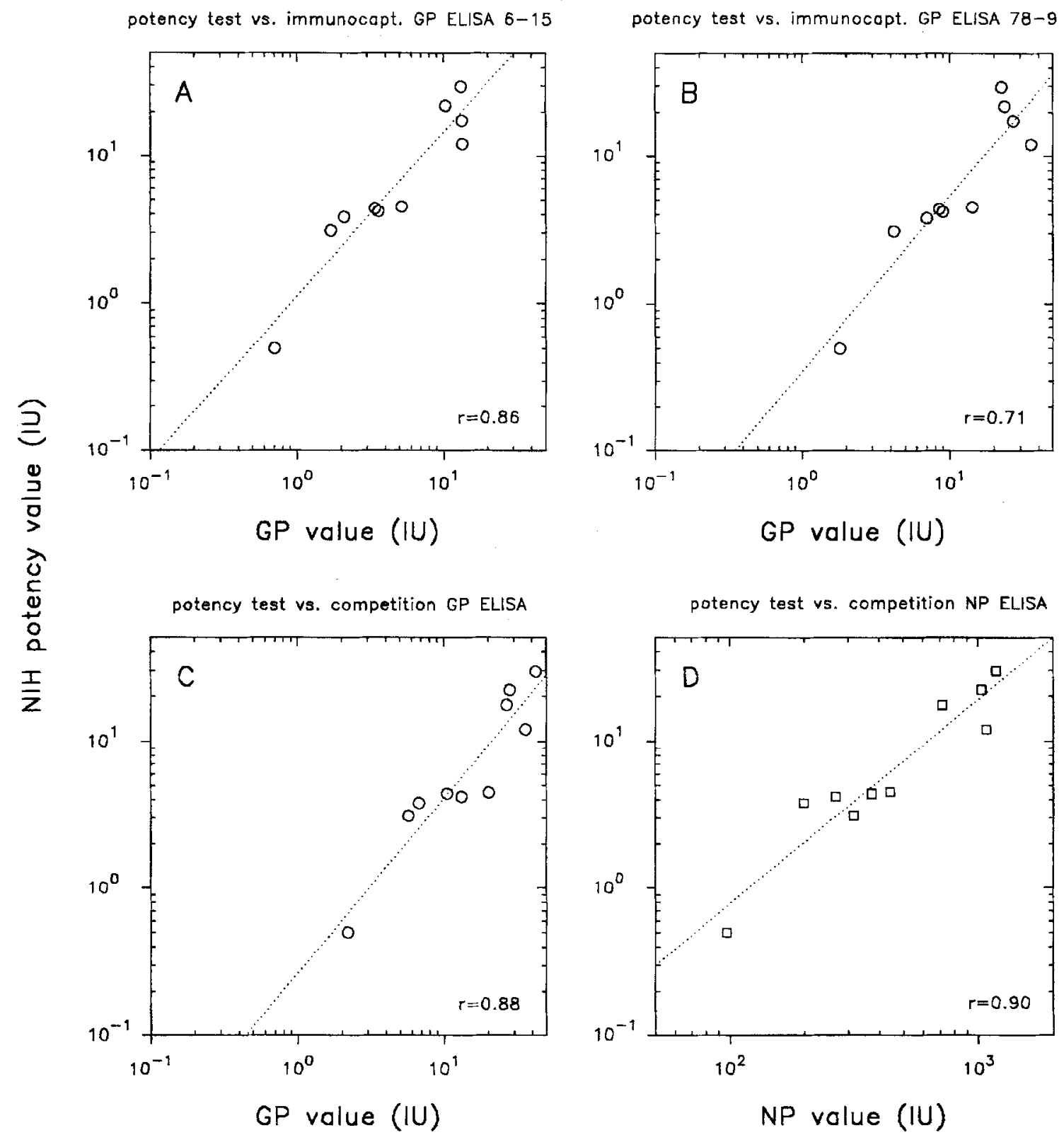

Fig. 1. Correlation between results in NIH potency test and different ELISAs for a panel of 10 human PM-DKC rabies vaccines. (A,B), immunocapture GP ELISAs 6-15 and 78-9 (sensitivity 0.03 and 0.05 GP units per dose, respectively); (C), competition GP ELISA (sensitivity 0.05 GP units per dose); (D), competition NP ELISA (sensitivity 0.8 NP units per dose). Correlation coefficients (r) and the regression lines (dotted line) were determined by linear regression analysis and are indicated in the graphs.

In the present study, we demonstrated the suitability of MAb-based ELISA techniques for quantitation of GP and NP contents in rabies vaccines. A good correlation (up to $r=0.88$ ) was shown between potency values on the one hand and GP values by immunocapture and competition ELISAs on the other hand, when PM-DKC vaccines were tested. Also good correlation 


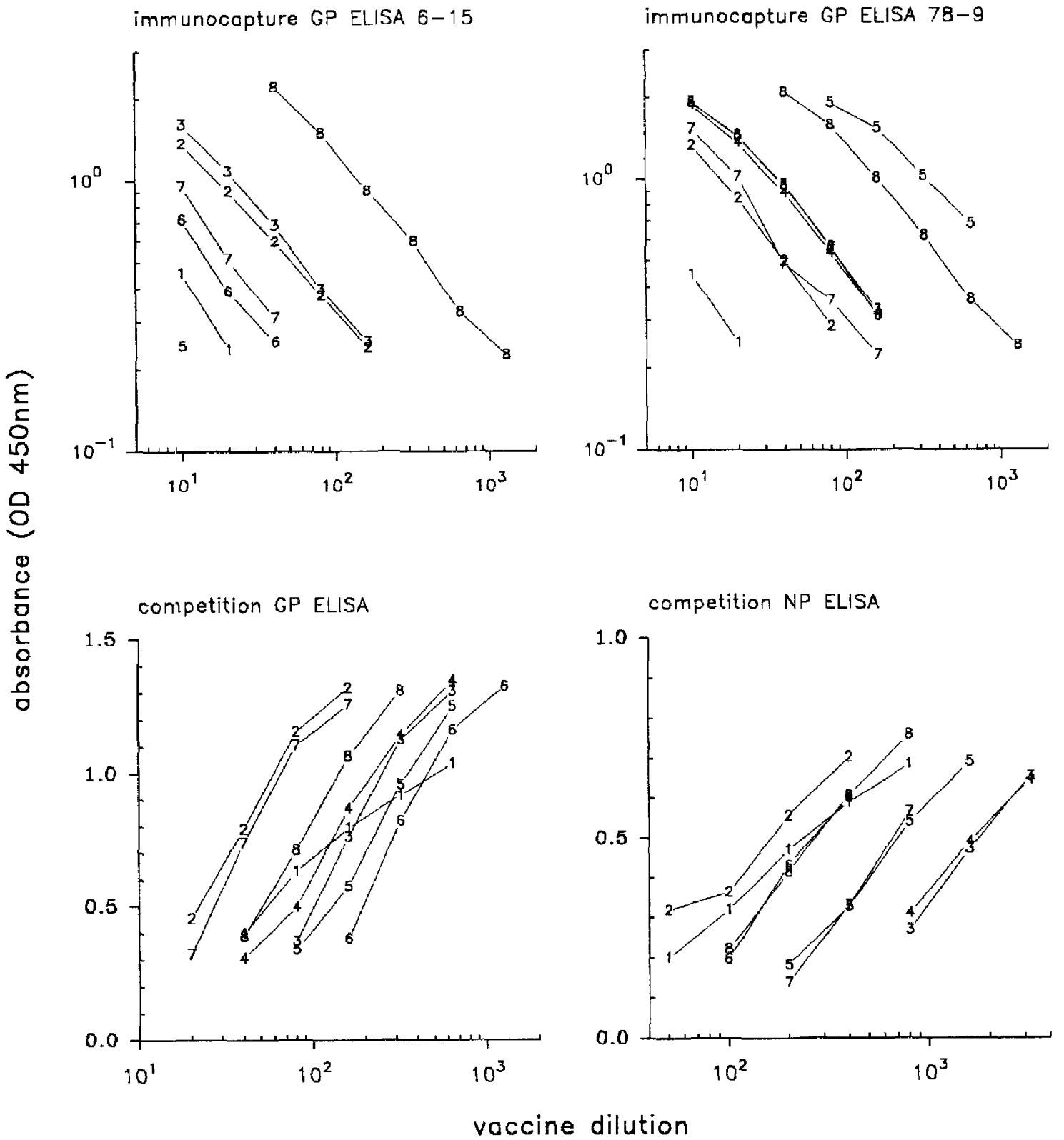

Fig. 2. Titration of veterinary rabies vaccines in immunocapture GP ELISAs 6-15 and 78-9, competition GP ELISA and competition NP ELISA. The panel of vaccines consisted of seven commercial veterinary rabies vaccines (no.1, SAD polymer adjuvanted; nos. 2 and 7, SAD; no.3, PM; no.4, LEP; nos.5 and 6, PV) and the International Standard for rabies vaccine (no.8, PM).

$(r=0.90)$ was found between potency values and NP values by competition NP ELISA for PMDKC vaccines. Furthermore, we demonstrated differences of applicability of GP ELISAs to veterinary rabies vaccines produced with PM, PV,
SAD and LEP vaccine strains. First, immunocapture GP ELISA 6-15 could not be applied to the LEP and one of the PV rabies vaccines, which is reasonably related to strain-specific differences in antigenic sites on the rabies virus GP. Second, 
although immunocapture GP ELISA 78-9 and competition GP ELISA using MAb 2-22 could readily be applied to the different vaccinc strains tested here, the results of the vaccine titrations by the ELISAs appeared to be different. These findings indicate that a product-specific reference vaccine is probably needed to calibrate the systems. Also special attention should be paid to adjuvants used in veterinary vaccines. The present data show that the results in ELISA for one of the SAD vaccines were invalid, which is likely caused by the presence of a polymer-based adjuvant.

Taken together, it was shown that in vitro GP and NP antigenicity control by ELISA has the potential to replace, at least in part, the mouse potency test for inactivated rabies vaccines. We suggest a further evaluation of the potential of the newly developed competition ELISAs in wellfounded collaborative studies. A rapid start with collaborative testing can be made with retrospective testing of already released rabies vaccine lots by the proposed ELISAs. It should be realized that antigenicity of vaccines as measured by ELISA systems, can only provide an indication of the potency in vivo. Additional support for the introduction of in vitro antigenicity ELISA systems, may be based on the simultaneous introduction of in vitro assays demonstrating antigenicity at the $T$ cell level in proliferative $T$ cell responses. PM-DKC vaccine induced proliferation of lymphoid cells from rabies vaccinated individuals showed good correlation with in vivo potency values and GP values in the competition ELISA presented here (Rooijakkers et al., 1995). Also assessment of virus neutralizing-Abs after rabies vaccination by a serological assay system, which is an important parameter for protection against rabies infection (Wunderli et al., 1991), should be carried out in the evaluation of alternative methods for the mouse potency test.

In conclusion, the introduction of alternative in vitro assay systems to estimate the potency of vaccines may only be considered if the production methods of the vaccine are well standardized, a good correlation with protective im- munity has been shown and the ELISA systems are properly validated.

\section{Acknowledgements}

The authors would like to thank Mr. A.A. Vermeulen, Mr. R.W.M. van Kinderen, Mr. P.W. Dissel and Mr. C. van Essen of the Central Animal Laboratory (CDL) for technical assistance, Dr. R. Hoenderken, veterinary inspector (VI) for collecting rabies vaccines and Ms. C. Kruyssen for assistance in preparing the manuscript. This work was supported by grants from the Dutch Platform Alternatieven voor Dierproeven (PAD 90-61) and the EC Programme on Alternatives to Animal Testings (B4-3081/91/007945).

\section{References}

Atanasiu, P., Perrin, P. and Delagneau, J.F. (1980) Use of an enzyme immunoassay with protein $A$ for rabies antigen and antibody determinations. Dev. Biol. Stand. 46, 207215.

Barth, R., Diderrich, G. and Weinmann, E. (1988) NIH test, a problematic method for testing potency of inactivated rabies vaccine. Vaccine 6, 369-377.

Barth, R., Gross-Albenhausen, E., Jaeger, O. and Milcke, L. (1981) The antibody-binding test: a useful method for quantitative determination of inactivated rabies virus antigen. J. Biol. Stand. 9, 81-89.

Barth, R., Franke, V., Müller, H. and Weinmann, E. (1990) Purified chick-embryo-cell (PCEC) rabies vaccine: its potency performance in different test systems and in humans. Vaccine 8, 41-48.

Bayer, E.A. and Wilchek, M. (1980) The use of avidin-biotin complex as a tool in molecular biology. Methods Biochem. Anal. 26, 1-45.

Bliss, C.I. (1935) The calculation of the dose-mortality curve. Ann. Appl. Biol. 22, 134-167.

Bunschoten, H., Gore, M., Claassen, I.J.Th.M., UytdeHaag, F.G.C.M., Dietzschold, B., Wunner, W.H. and Osterhaus, A.D.M.E. (1989) Characterization of a new virus-neutralizing epitope that denotes a sequential determinant on the rabies virus glycoprotein. J. Gen. Virol. 70, 291-298.

Dietzschold, B., Gore, M., Casali, P., Ueki, Y., Rupprecht, C.E., Notkins, A.L. and Koprowski, H. (1990) Biological characterization of human monoclonal antibodies to rabies virus. J. Virol. 64, 3087-3090.

European Pharmacopoeia (1985) Vaccinum rabiei inactivatum ad usum veterinarium. In: Monograph European Pharmacopoeia. 2nd Edn, part II/9, 451 . 
Ferguson, M. and Heath, A. (1992) Report of a collaborative study to assess the determination of glycoprotein antigen content of rabies vaccines for human use. Biologicals 20 , 143-154.

Ferguson, M. and Schild, G.C. (1982) A single radial immunodiffusion technique for the assay of rabies glycoprotein antigen: application for potency tests of vaccines against rabies. J. Gen. Virol. 59, 197-201.

Ferguson, M., Seagroatt, V. and Schild, G. (1984) A collaborative study on the use of single radial immunodiffusion for the assay of rabies virus glycoprotein. J. Biol. Stand. 12, 283-294.

Groen, J., Van der Groen, G., Hoofd, G. and Osterhaus, A.D.M.E. (1989) Comparison of immunofluorescence and enzyme-linked immunosorbent assays for the serology of hantavirus infections. J. Virol. Meth. 23, 195-203.

Hendriksen, C.F.M., Slob, W., Van der Gun, J.W., Westendorp, J.H.L., Den Bieman, M., Hesp, A. and Van Zutphen, L.F.M. (1994) Immunogenicity testing of diphtheria and tetanus vaccines by using isogenic mice with possible implications for potency testing. Lab. Anim. 28, 121-129.

Hong, H.A., Rooijakkers, E.J.M., Ke, N.T., Groen, J. and Osterhaus, A.D.M.J. (1994) Methods for the purification of equine rabies immunoglobulin: effects on the yield and biological activity. Biologicals 22, 1-6.

Lafon, M., Perrin, P., Versmisse, P. and Sureau, P. (1985) Use of monoclonal antibody for quantitation of rabies vaccine glycoprotein by enzyme immunoassay. J. Biol. Stand. 13, 295-301.

Lorenz, R.J. and Bögel, K. (1973) Methods of calculation. In: M.M. Kaplan and H. Koprowski (Eds.), Laboratory Techniques in Rabies, 3rd Edn., World Health Organization, Geneva, pp. 321-335.

Lyng, J., Wics Bentzon, M., Ferguson, M. and Fitzgerald, E.A. (1992) Rabies vaccine standardization: International collaborative study for the characterization of the fifth International Standard for rabies vaccine. Biologicals 20 , 301-313.

Nakane, P.K. and Kawaoi, A. (1974) Peroxidase-labeled antibody. A new method of conjugation. J. Histochem. Cy. tochem. 22, 1084-1091.

Osterhaus, A.D.M.E., Groen, J., UytdeHaag, F.G.C.M., Bunschoten, E.J., De Groot, I.G.M., Van der Meer, R. and Van Steenis, G. (1990) Quantification of rabies virus vaccine glycoprotein by ELISA with monoclonal antibodies: correlation with single radial diffusion and the induction of anti-viral antibodies and protection in mice. In: O. Thraenhart, H. Koprowski, K. Bögel and P. Sureau (Eds.), Progress in Rabies Control, Staples Printers Rochester Ltd., Rochester, pp. 320-323.

Osterhaus, A.D.M.E., Van Wezel, A.L., Van Steenis, G., Hazendonk, A.G. and Drost, G.A., (1982) Production and potential use of monoclonal antibodies against polioviruses. Dev. Biol. Stand. 50, 221-223.

Perrin, P., Morgeaux, S. and Sureau, P. (1990) In vitro rabies vaccine potency appraisal by ELISA: Advantages of the immunocapture method with a neutralizing ant1-glycoprotein monoclonal antibody. Biologicals 18, 321-330.

Rooijakkers, E.J.M., Groen, J., Uittenbogaard, J.P., Van Herwijnen, J.R. and Osterhaus, A.D.M.E. (1995) Development and evaluation of alternative testing methods for the in vivo NIH potency test used for the quality control of inactivated rabies vaccines. Dev. Biol. Stand. 86, 137-145.

Seligmann, E.B. Jr. (1973) The NIH test for potency. In: M.M. Kaplan and H. Koprowski (Eds.), Laboratory Techniques in Rabies. 3rd Edn., World Health Organization, Geneva, pp. $279-286$.

Sureau, P. (1992) Contribution to rabies prevention. Vaccine $10,896-899$.

Thraenhart, O. and Ramakrishnan, K. (1989) Standardization of an enzyme immunoassay for the in vitro potency assay of inactivated tissue culture rabies vaccines: determination of the rabies virus glycoprotein with polyclonal antisera. J. Biol. Stand. 17, 291-301.

Thraenhart, O. and Ramakrishnan, K. (1990) Essen-ELISA, a standardized method for the potency assay of rabies vaccines and in process preparations. In: $\mathbf{O}$. Thraenhart, $\mathrm{H}$. Koprowski, K. Bögel and P. Sureau (Eds.), Progress in Rabies Control, pp. 324-345, Staples Printers Rochester Ltd., Rochester.

Van der Marel, P. and Van Wezel, A.L. (1981) Quantitative determination of rabies antigen by ELISA. Dev. Biol. Stand. 50, 267-275.

WHO (1992) WHO Expert Committee on Rabies, 8th report. WHO Technical Report Series 824, 1-84.

Wunderli, P.S., Shaddock, J.H., Schmid, D.S., Miller, T.J. and Baer, G.M. (1991) The protective role of humoral neutralizing antibody in the NIH potency test for rabies vaccines. Vaccine 9, 638-642. 